\title{
Nanoswimmer-oriented Direct Targeting Strategy Inspired by Momentum-based Gradient Optimization
}

\author{
Muhammad Ali ${ }^{1}$, Michael J. Cree ${ }^{2}$, Neda Sharifi ${ }^{3}$, Yifan Chen $^{4}$
}

\begin{abstract}
This paper considers the advantage of knowledgeaided direct targeting strategy (DTS) over systemic targeting for tumor homing in complex human vasculature. Tumor location in the body can be estimated by closely observing the tumortriggered bio-physical gradients in its vicinity, helping drugloaded magnetic nanoswimmers to accumulate at the disease location. These nanoswimmers are assembled by magnetic nanoparticles (MNPs), which act as contrast agents increasing the diagnostic capability of different medical imaging techniques. We propose a novel DTS inspired by the iterative gradient descent (GD) with momentum optimization for tumor targeting amplification. We show by computational experiments that the MNPs accumulate at the disease location at a faster rate and the probability of tumor detection is higher for the proposed knowledge-aided DTS as compared to the knowledgeless systemic targeting. We believe that our work for tumor targeting amplification based on such nanosystem will open new horizons in the field of diagnosing tumor at its early stage.

Index Terms: Direct targeting strategy, touchable smart nanosystem, tumor-triggered biophysical gradients, medical imaging techniques.
\end{abstract}

\section{INTRODUCTION}

Cancer is one of the biggest causes of mortalities globally. Different medical imaging techniques (MITs) are routinely used to locate tumors in the human body. It is important to locate the tumor as early as possible when its size is very small, however resolution capability of these MITs is not sufficient for the purpose [1]. This leads to the strategy of contrast enhanced medical imaging where magnetic nanoparticle (MNP) assembled nanoswimmers can be used as contrast agents. These nanoswimmers once attached to the tumor, highlight the disease area for MRI and microwave imaging.

Knowledge-less systemic targeting can deliver a very small fraction of nanoparticles to tumor location [2]. It is because of their dependence on systemic circulation, nonavailability of sensory based displacement capability to target hypoxic regions, and insufficient propelling force. Moreover, nanoswimmers tend to consume inside the human vasculature due to branching (choosing wrong vessel), diffusion (spreading), and degradation (deterioration). Direct targeting strategy (DTS) can be used to increase the deposition of contrast agents to the disease. External manipulability of the nanoswimmers is achieved by applying a constant magnetic

*Asterisk indicates corresponding author.

${ }^{1} \mathrm{M}$. Ali , ${ }^{2} \mathrm{M}$. J. Cree and ${ }^{3} \mathrm{~N}$. Sharifi are with the School of Engineering, University of Waikato, Hamilton, New Zealand. (ma316@students.waikato.ac.nz)

${ }^{*} \mathrm{Y}$. Chen is with the School of Engineering and the School of Computing and Mathematics, University of Waikato, Hamilton, New Zealand. (yifan.chen@waikato.ac.nz)

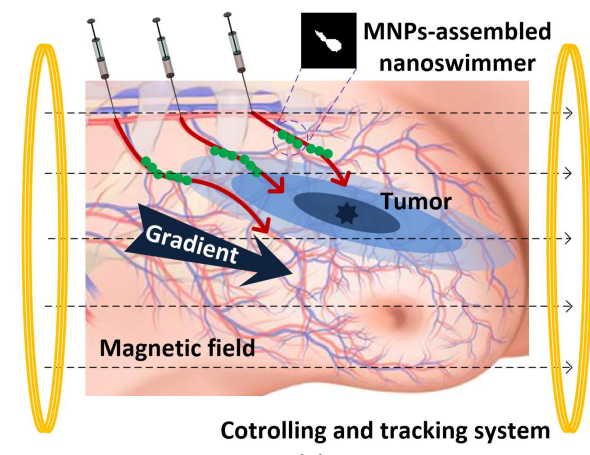

(a)

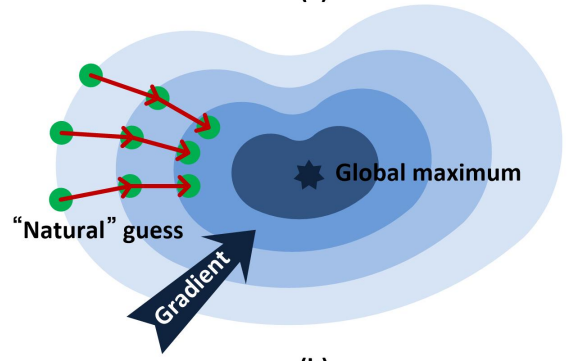

(b)

Fig. 1. Analogy between (a) DTS employed in externally manipulable smart nanosystem for tumor sensitization and (b) Iterative optimization process.

field which makes sure that nanoswimmers adopt the shortest path and encounter minimum systemic exposure. Knowledge of the location of the tumor helps to externally track and control nanoswimmers and direct them towards the tumor as compared to knowledge-less systemic targeting. Hence, under the influence of external magnetic field, a large accumulation of $\mathrm{MC}-1$ cells, roughly $55 \%$ of drug containing nanoswimmers reach the center of the tumor [3].

Tumor homing can also be amplified with the help of the so-called smart nanosystems in which the living host environment is used to assist with the detection of the tumor [4]. These smart nanosystems can be divided in two main categories: environment responsive and environment primed [5]. The first category comprises of nanoparticles that sense and respond to their environment. The second category refers to schemes in which a host environment is administered by external influences such as x-rays, heat, drugs, infrared light or nanoparticles themselves [6]. However, these smart nanosystems have no knowledge about the tumor location and rely on systemic targeting for tumor sensitization.

We can harness many advantages from knowledge-aided externally manipulable nanoswimmers for direct targeting 


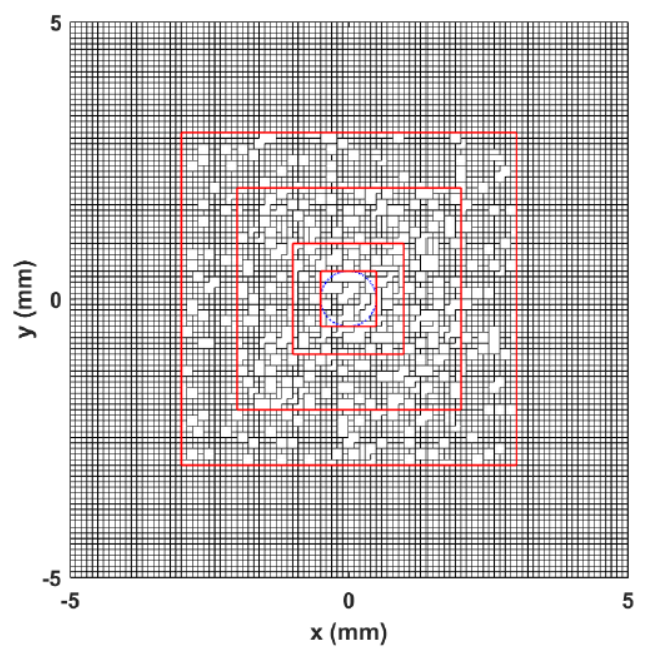

Fig. 2. Simulated multi-layer vascular network. The level of occupancy on the lattice reduces from $100 \%$ to $40 \%$ gradually as the distance to the tumor center decreases. The boundaries of the layers are denoted by the red solid lines. The tumor center is denoted by the blue dotted circle.

such as having minimum systemic exposure using the shortest physiological path with external manipulation but it cannot work in an adequate manner without knowing the location of tumor. On the other hand, knowledge-less smart nanosystems cannot be externally manipulable thus do not guarantee to reach tumor using smallest distance with least exposure to the environment. So the idea is to combine the desired features of both systems into a new system which can be termed as touchable smart nanosystem where we can use bio-physical gradients to guide the direct targeting process and formulate it as an optimization problem.

The paper is organized as follow. In section II, analogy between our proposed touchable smart nanosystem and iterative optimization process has been explained along with discretized vascular network model. Section III explains the objective function required to represent bio-physical gradients and computational framework followed by performance analysis in section IV. Finally some concluding remarks are given in section $\mathrm{V}$.

\section{DIRECT DRUG TARGETING AS NATURAL COMPUTING}

\section{A. Analogy between Externally Manipulable Smart Nanosys- tem and Iterative Optimization}

Biological gradients can be exploited from the physical properties of the host environment such as peritumoral vasculature architecture and blood flow velocity. Similarly, host environment can also be primed to induce bio-physical gradients. Gold nanorods, for example, are modified to circulate in blood for longer periods of time and passively accumulate in tumors. They are used to heat tumor tissues by amplifying the absorption of near-infrared energy that is mostly tranparent to living tissues. As a result of this hyperthermia, gradient of blood flow velocity is amplified due to the difference in response of normal and tumor microcirculation.
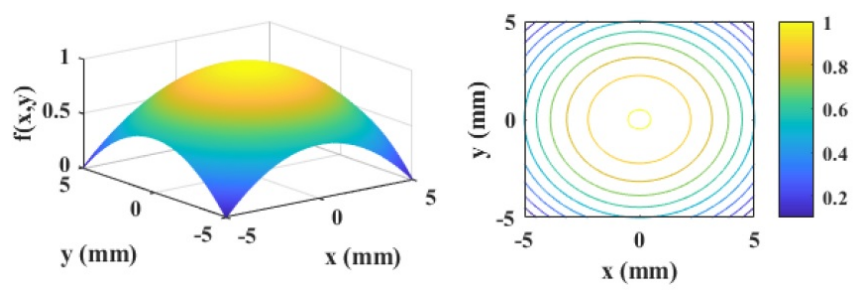

Fig. 3. Illustration of $\mathrm{f}(\mathrm{x}, \mathrm{y})$ for sphere function and its contour plot

There is a fascinating analogy between the proposed externally manipulable smart nanosystem and iterative optimization as shown in Fig. 1. Firstly, the global maximum of an objective function in iterative optimization corresponds to the epicenter of the tumor. Secondly, the domain of the objective function corresponds to the tissue region under observation or region of interest (ROI). Similarly, guess inputs that are made in iterative optimization are the swarm of nanoswimmers in smart nanosystem.

\section{B. Multilayer Vascular Network Model}

Microvascular density is more in the peritumoral region as compared to the center of the tumor. Moreover, normal tissues are regularly vascularized, which result in homogeneous lattice comprised of straight and rigid cylindrical capillaries joining adjacant nodes whereas tumor vasculature is very complex in nature [7] and require an invasion percolation method to explain its higher fractal dimesions. Keeping these properties in mind, we have developed a model for the possible growth of vasculature around the tumor which is implemented by first assigning uniformly distributed random values of strength to each point on the underlying square lattice. Then the network is populated by occupying the lattice point adjacent to current location (which was a random starting point) having minimum strength. The process is repeated until a required occupancy level is achieved. All adjacent occupied points are connected by the blood vessels. Then, a multilayer vascular network has been developed to depict the fuzzy and blurred boundaries of malignant tumors [8]. This discretized model is required to quantify the diffusive nature of tissue anomaly which take care of the gradual and continuous change in the fractal dimensions across the tumor.

\section{FORMULATION OF PROPOSED DTS}

\section{A. Objective Function}

In the current work, tumor homing is achieved indirectly through an external controlling and tracking system as shown in Fig. 1(a). It is necessary that the in-vivo biological gradients should be mapped to an externally measurable objective function by using nanoswimmers as a probe for analysis of the host environment. Let $f$ represent the externally measurable objective function be defined on the domain $\mathbb{D}$, which represent the high risk tissue under observation. We have taken the sphere function to evaluate the performance of touchable smartnanosystem as shown in Fig. 3. Its landscape 


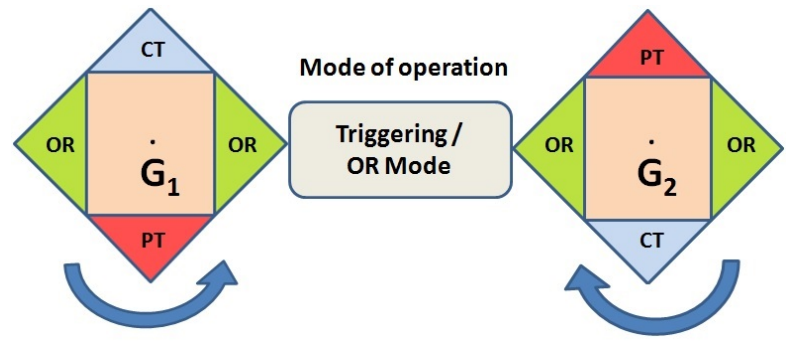

Fig. 4. Proposed framework where $G_{1}$ and $G_{2}$ take turn to operate in planned triggering (PT) and coupled triggering (CT) modes with a common oberving and recording (OR) mode. Rectangle between them represents the current mode of operation they are in at any particular instant.

represents the gradual change in gradients in the peritumoral region. The maximum value is normalized to 1 and the minimum value is 0 . The search domain is $-5 \mathrm{~mm} \leq x, y \leq$ $5 \mathrm{~mm}$. The landscape of the bowl-shaped sphere function is

$$
\begin{aligned}
& f(x, y)= \\
& \left\{\begin{array}{l}
1, \\
1-0.02\left(x^{2}+y^{2}\right) \\
\sqrt{x^{2}+y^{2}}>0.5 \text { and }(x, y) \in \mathbb{V} .
\end{array}\right.
\end{aligned}
$$

The term $\mathbb{V}$ denotes the discrete vascular network shown in Fig. 2. The sphere function is a convex function with no local maxima except the global one. The tumor center is denoted by a small circle of radius $0.5 \mathrm{~mm}$ located at the origin which represent the highest values of $f(x, y)$. For simplicity, we have just imprinted the objective function in (1) on the vascular network $\mathbb{V}$.

\section{B. Proposed Framework}

In the computational framework, for simplicity, we suppose two guess inputs $G_{1}$ and $G_{2}$ which are deployed at the sites $\mathbb{R}_{1}$ and $\mathbb{R}_{2} \subseteq \mathbb{D}$ where $\mathbb{R}_{1}$ and $\mathbb{R}_{2}$ denote the injection sites and $\mathbb{D}$ is the domain of region of interest as shown in Fig. 1. The switching between modes of operation for both guesses is shown by the big boxes moving anticlockwise (for $G_{1}$ ) and clockwise (for $G_{2}$ ) in the direction of arrows in Fig. 4. They operate in three modes: planned triggering (PT), observing and recording (OR) and coupled triggering (CT) as explained below:

1) Initialization: Before the guesses start to operate in any mode, they are deployed at the same time so that they can start searching for the optimal solution based on an iterative algorithm explained in Section III-C.

2) OR Mode: After injection or initialization, both guesses operate in the common OR mode. It is in this mode that the area around each guess is explored to look for the maximum gradient. This corresponds to nanoswimmers moving in natural environment in the absence of any external magnetic field, and their movements are observed and recoded by the external monitoring system for PT and CT modes explained next.

3) PT Mode: After the common $\mathrm{OR}$ mode, moving in the directions of arrows in Fig. $4, G_{1}$ goes in the PT mode whereas $G_{2}$ goes in the CT mode. In PT mode, depending upon the gradients calculated in the OR mode, $G_{1}$ takes a step toward the maximum gradient direction that will most probably lead to the optimal solution (tumor). This corresponds to application of the external magnetic field to manoeuvre nanoswimmers towards the location of the tumor under the guidance of the bio-physical gradient.

4) CT Mode: When $G_{1}$ is in PT mode, $G_{2}$ is in CT mode, so it goes in the same direction where $G_{1}$ is moved because its movement is coupled with the movement of $G_{1}$. This corresponds to the situation that the same magnetic field is present for both swarms of nanoswimmers, which can control only one swarm at a particular time. After the next common OR mode, when $G_{2}$ goes in the PT mode, $G_{1}$ will operate in the CT mode with its movement now coupled with the movement of $G_{2}$. Hence, both guesses take turn to operate in PT and CT modes while having the common OR mode in between.

\section{DTS Inspired by Momentum-based GD}

The momentum based GD-inspired DTS starts with a generic guess $G_{1}$ located at $\vec{x}_{1}$ which attempts to find a global maximum $f\left(\vec{x}^{*}\right)$. For the framework shown in Fig. 4, consider the sequence $\vec{x}_{1}\left(O R_{1}^{(1)}\right), \vec{x}_{1}\left(O R_{2}^{(1)}\right), \vec{x}_{1}\left(O R_{3}^{(1)}\right), \cdots, \vec{x}_{1}\left(O R_{\mathrm{N}}^{(1)}\right)$ representing the positions of $G_{1}$ observed by the external system in the first OR mode through $N$ OR processes represented by a cluster in Fig. 5. In the classical GD, for location update, we have

$$
\begin{array}{r}
\vec{x}_{1}\left(O R_{\mathrm{m}}^{(1)}\right)=\vec{x}_{1}\left(O R_{\mathrm{m}-1}^{(1)}\right)+\gamma_{m-1} \nabla f \vec{x}_{1}\left(O R_{\mathrm{m}-1}^{(1)}\right) \\
\text { where } m=2,3, \cdots, N
\end{array}
$$

However, in our case, where location updating is interrupted by multiple OR and CT processes, the position update is modified as

$$
\vec{x}_{1}\left(O R_{\mathrm{m}}^{(1)}\right)=\vec{x}_{1}\left(P T^{(1)}\right)+\gamma_{m} \nabla f \vec{x}_{1}\left(P T^{(1)}\right)
$$

The gradient $\nabla f \vec{x}_{1}\left(P T^{(1)}\right)$ is estimated through $N$ OR processes. If the gradient is not changing much from $O R_{\mathrm{m}}^{(1)}$ to $O R_{\mathrm{m}+1}^{(1)}$, it can be estimated as

$$
\nabla f \vec{x}_{1}\left(P T^{(1)}\right) \approx \max _{m=1,2, \cdots, N}\left\{\nabla f\left(\vec{x}_{1}\left(O R_{\mathrm{m}}^{(1)}\right)\right)\right\}
$$

Otherwise, only the final gradient estimate is used such that

$$
\nabla f \vec{x}_{1}\left(P T^{(1)}\right) \approx\left\{\nabla f\left(\vec{x}_{1}\left(O R_{\mathrm{N}}^{(1)}\right)\right)\right\}
$$

Suppose that $f(\vec{x})$ is convex and $\nabla f(\vec{x})$ is Lipshitz, the step size $\gamma_{m}$ can be chosen to guarantee convergence to a global optimum by using the Brazilai-Borwein method [9]. Finally momentum is introduced to help accelerate GD in the relevant direction and dampen oscillations. It does this 


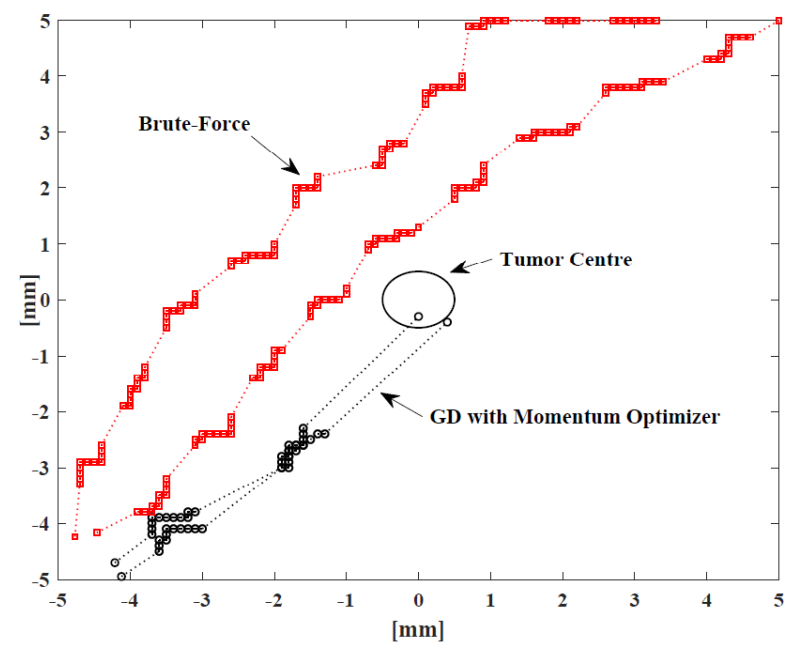

Fig. 5. Trajectories of guess inputs when proposed framework is applied: “०” - Momentum based GD-inspired DTS, “ $\square$ " - brute-force search.

by adding a momentum term $\alpha$ of the update vector $\beta_{i-1}$ of the past time step to the current update vector $\beta_{i}$ as

$$
\begin{aligned}
& \beta_{i}=\alpha \beta_{i-1}+\gamma_{m} \nabla f \vec{x}_{1}\left(P T^{(1)}\right), \\
& \quad \text { and } \vec{x}_{1}\left(P T^{(1)}\right)=\vec{x}_{1}\left(P T^{(1)}\right)+\beta_{i}
\end{aligned}
$$

The momentum term $\alpha$ is set to be 0.9 . In our case, when using momentum, we push our guess in the direction of maximum gradient. The step size for PT mode increases for dimensions whose gradients point in the same directions and reduces step size for dimensions whose gradients change directions. This results in faster convergence to the global maximum (i.e. tumor location) with reduced oscillations.

\section{PERFORMANCE ANALYSIS}

We used MATLAB as a simulation tool to evaluate performance of the momentum based GD-inspired DTS and compared it with brute-force search. The maximum search time allowed was set to be 200s for each of the 200 simulations. For the DTS, the searching process was stopped if any of the guess reached the cancer center assuming that the other guess would be guided to the tumor center if it had not overshot the tumor location. For the brute-force search, each guess followed random walk in the lattice as compared to the DTS, which followed the framework outlined in Section III-B.

Fig. 5 shows the typical trajectories of guess inputs for the landscape of sphere function when both the proposed momentum based GD-inspired DTS and the brute- force search are considered. As a result, the proposed DTS successfully detects the tumor center whereas the brute-force search fails to do so. Fig. 6 represents the histograms of search time, which shows that our proposed DTS detects the tumor in less time because the current step taken by the guess considers the direction of the previous steps taken by it. If it finds that the direction is correct (i.e. towards the global maximum), it increases its step size resulting in faster target detection.

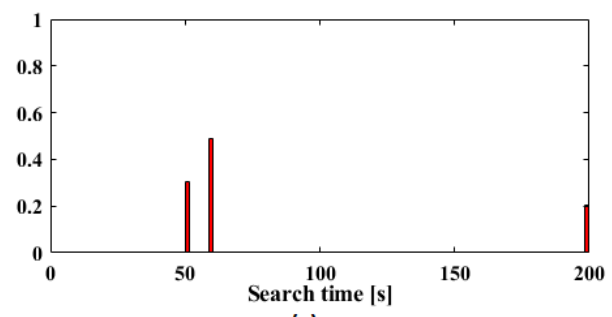

(a)

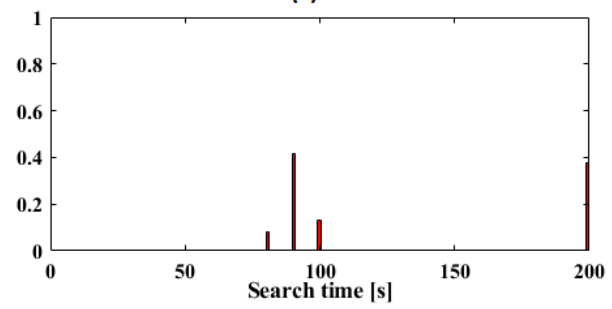

(b)

Fig. 6. Histograms of search time when: (a) DTS inspired by Momentumbased GD and (b) brute-force search are applied in a sphere landscape.

\section{CONCLUSIONS}

We have proposed a novel momentum based GD-inspired DTS for smart nanosystems. We have shown by computational experiments that proposed DTS localized the tumor faster as compared to brute-force search. In future, proposed framework may be applied to other objective functions like Easom or Matyas functions representing more complex landscapes. Finally, proposed DTS strategy and objective function should be validated by real experiments to justify clinical relevance of proposed strategy.

\section{REFERENCES}

[1] H. Kasban, M. El-Bendary, and D. Salama, "A comparative study of medical imaging techniques," Int. J. Information Sci. Intelligent System, vol. 4, pp. 37-58, 2015.

[2] Y. H. Bae and K. Park, "Targeted drug delivery to tumors: myths, reality and possibility," Journal of controlled release, vol. 153, no. 3, p. 198, 2011.

[3] O. Felfoul, M. Mohammadi, S. Taherkhani, D. De Lanauze, Y. Z. Xu, D. Loghin, S. Essa, S. Jancik, D. Houle, M. Lafleur et al., "Magnetoaerotactic bacteria deliver drug-containing nanoliposomes to tumour hypoxic regions," Nature nanotechnology, vol. 11, no. 11, p. 941, 2016.

[4] K. Seidi, H. A. Neubauer, R. Moriggl, R. Jahanban-Esfahlan, and T. Javaheri, "Tumor target amplification: Implications for nano drug delivery systems," Journal of controlled release, 2018.

[5] E. J. Kwon, J. H. Lo, and S. N. Bhatia, "Smart nanosystems: Bioinspired technologies that interact with the host environment," Proceedings of the National Academy of Sciences, vol. 112, no. 47, pp. 14 460-14 466, 2015.

[6] G. Von Maltzahn, J.-H. Park, K. Y. Lin, N. Singh, C. Schwöppe, R. Mesters, W. E. Berdel, E. Ruoslahti, M. J. Sailor, and S. N. Bhatia, "Nanoparticles that communicate in vivo to amplify tumour targeting," Nature materials, vol. 10, no. 7, p. 545, 2011.

[7] Y. Gazit, D. A. Berk, M. Leunig, L. T. Baxter, and R. K. Jain, "Scaleinvariant behavior and vascular network formation in normal and tumor tissue," Physical review letters, vol. 75, no. 12, p. 2428, 1995.

[8] R. M. Rangayyan, N. M. El-Faramawy, J. L. Desautels, and O. A. Alim, "Measures of acutance and shape for classification of breast tumors," IEEE Transactions on medical imaging, vol. 16, no. 6, pp. 799-810, 1997.

[9] J. Barzilai and J. M. Borwein, "Two-point step size gradient methods," IMA journal of numerical analysis, vol. 8, no. 1, pp. 141-148, 1988. 